\title{
Stathmin and EGFR correlates to HPV status and clinical outcome in sinonasal inverted papilloma*
}

\author{
Alexandra Elliot ${ }^{1}$, Anders Näsman², Marit Westman³ ${ }^{3}$ Lalle Hammarstedt- \\ Nordenvall' , Pär Stjärne'1, Linda Marklund' \\ ' Department of Clinical Science, Intervention and Technology, Division of ENT Diseases, Karolinska Institutet, Stockholm, \\ Sweden \\ ${ }^{2}$ Department of Oncology-Pathology, Department of Clinical Pathology, Cancer Center Karolinska, Karolinska Institutet, \\ Stockholm, Sweden \\ ${ }^{3}$ Department of Medicine Solna, Immunology and Allergy Unit, Karolinska Institutet, Stockholm
}

Rhinology 58: 1, 74 - 79, 2020

https://doi.org/10.4193/Rhin19.078

*Received for publication:

January 20, 2019

Accepted: August 7, 2019

\begin{abstract}
Background: Inverted papilloma (IP) is a locally destructive benign tumour of the sinonasal mucosa with a tendency for malignant transformation. Stathmin and epidermal growth factor receptor (EGFR) are important markers in cancer prognosis. Here we investigate if expression of stathmin and EGFR correlate to dysplasia, recurrence and HPV in IP.
\end{abstract}

Methods: 98 patients with IP diagnosed 2000-2010 were analyzed for stathmin and EGFR by immunohistochemistry (IHC) and HPV by polymerase chain reaction assay (PCR).

Results: All IPs expressed stathmin while its expression was absent or weak in normal mucosa. Dysplasia was present in $26,7 \%$ of IPs with high stathmin expression while only $7.4 \%$ of IPs with low stathmin expression showed dysplasia. Stathmin positive IPs showed a trend towards earlier recurrences. 57.1\% of IP expressed EGFR but no significant association was seen between EGFR-positivity and recurrence or dysplasia. EGFR was expressed by $91.7 \%$ of the HPV-positive IPs compared to $52,3 \%$ of the HPVnegative IPs.

Conclusions: EGFR expression is significantly higher in HPV positive IP. Stathmin is expressed by all IP tumour cells. Stathmin was also associated with dysplasia and a trend towards a correlation between stathmin positivity and recurrence was found. Stathmin and EGFR might therefore be considered therapeutic targets.

Key words: EGFR, HPV, inverted papilloma, outcome, stathmin

\section{Introduction}

Inverted papilloma (IP) is a locally destructive benign tumour of the sinonasal mucosa with a tendency for malignant transformation ${ }^{(1,2)}$. The aetiology is mainly unknown, although human papillomavirus (HPV) has been suggested by some authors as an aetiological factor in a subset of these tumours ${ }^{(3)}$. There is also a lack of knowledge of molecular prognostic factors in IP and a lack of knowledge of which patients that may relapse.

Expression of onco-proteins is a relatively new area of interest in research on IP. Stathmin, also called oncoprotein 18, is a protein crucial for the assembly and disassembly of the mitotic spindle during cell division ${ }^{(4)}$. The expression of stathmin has previously been shown to be associated with different cancers, among others oesophageal, urothelial and oral carcinomas ${ }^{(5-7)}$, and has been associated with poor prognosis. It has been suggested by others that HPV could be implicated in the pathogenesis of IP and that stathmin expression is elevated in IPs, correlates with HPV status and is higher expressed in recurrent cases and in IPS with higher Krouse stage ${ }^{(8)}$.

The epidermal growth factor receptor (EGFR) is involved in the pathogenesis and progression of different carcinomas ${ }^{(9,10)}$ and data indicates that EGFR plays a role in the development and prognosis of many head and neck squamous cell carcinomas (HNSCC) including sinonasal carcinomas ${ }^{(11,12)}$. It has also been 
proposed that up-regulation of EGFR could be correlated to dysplasia and malignant transformation of sinonasal IP ${ }^{(13,14)}$. It's unknown whether stathmin and EGFR interact or if there is any correlation between expression of the markers.

This study aims to investigate how expression of stathmin and EGFR correlates to dysplasia and recurrence in IP, alone and in combination with HPV.

\section{Subjects and methods}

Patient

Patients were identified from the Swedish Cancer Registry (SCR) and the study base consisted of patients $(n=126)$ diagnosed with IP in Stockholm between 2001 - 2010. Formalin fixed, paraffin embedded (FFPE) blocks with specimens of IP were retrieved from Stockholms Medicinska Biobank (SMB). After histological re-evaluation of the original diagnosis by a qualified pathologist, 98 cases out of 126 were obtained for further analysis.

Patient data (age at diagnosis, gender, recurrence data, followup time, and malignant transformation) were retrieved from the medical records and SCR. Reported surgical margins was obtained from the surgical notes. Cases with unsure margins or where the question of radicality was not mentioned were considered as positive surgical margins. Data on reported dysplasia were obtained from the histopathological reports.

The study was approved by the Regional Ethical Review Board at Karolinska Institutet, Stockholm, Sweden (2012/49-31/2).

\section{Detection of HPV DNA}

Presence of HPV DNA was analysed as previously described ${ }^{(15)}$. In brief, 2 x $15 \mathrm{~mm}$ sections from FFPE IP tissue blocks, with blank controls in parallel to detect cross-contamination, were cut and DNA was purified using the Roche High Pure FFPET DNA Isolation kit (Roche Diagnostics GmbH, Mannheim, Germany). Presence of HPV DNA was analysed with a multiplex LUMINEX assay covering 27 HPV types (HPV 6, 11, 16, 18, 26, 30, 31, 33, 35 , $39,42,43,44,45,51,52,53,56,58,59,66,67,68,69,70,73$ and

82 ) with the house keeping gene $\beta$-globin included as a positive control.

\section{EGFR and Stathmin immunohistochemistry}

EGFR and stathmin expression was analysed by immunohistochemistry on 4um FFPE slides, using the rabbit monoclonal antibodies, anti-EGF Receptor XP® (clone: D38B1, dilution: 1:50, Cell Signaling, Massachusetts, USA) and anti-Stathmin 1 RabMab $^{\circledast}$ (clone: EP1573Y, dilution: 1:250, Abcam, United Kingdom), respectively. A standard streptavidin-biotin peroxidase protocol was applied as previously described, with heat antigen retrieval in citrate buffer $(\mathrm{pH} 6)^{(16)}$.

Positive EGFR staining was defined as membranous staining with strong intensity and positive stathmin staining was defined
Table 1. Patient characteristics.

\begin{tabular}{|lc|}
\hline Characteristic & Value \\
\hline Age & \\
\hline Range & $18-89$ \\
\hline Mean & 58.0 \\
\hline Median & 59.5 \\
\hline Sex & \\
\hline Females & $30(30.6 \%)$ \\
\hline Males & $68(69.4 \%)$ \\
\hline HPV & $11(11.2 \%)$ \\
\hline Low risk HPV positive & $1(1.0 \%)$ \\
\hline High risk HPV positive & $86(87.6 \%)$ \\
\hline Negative & \\
\hline EGFR & $0-90$ \\
\hline Range & $56(57.1 \%)$ \\
\hline EGFR positive & $42(42.9 \%)$ \\
\hline EGFR negative & \\
\hline Stathmin & $10-100$ \\
\hline Range & $15(15.6 \%)$ \\
\hline Stathmin positive & $81(84.4 \%)$ \\
\hline Stathmin negative & \\
\hline
\end{tabular}

as a strong cytoplasmic staining. Fraction of EGFR and stathmin positive tumour cells (closest 10\%) was analysed by two independent researchers ( $A E$ and LM) blinded for any information on the tumour. Discrepant opinions (approximately in 10\% of cases) were solved by consensus.

\section{Statistical analysis}

The Pearson Chi-square and the Fisher exact test was used for categorical data and the Mann Whitney U-test was used outcomes that did not have a normal distribution. Recurrence free time was defined as time from diagnosis until a documented recurrence of disease. Patients lost during follow-up were censured. All patients were considered as tumour free after surgery, independent of reported surgical radicality. Patients without any follow-up were censored day 0 . The Kaplan-Meier estimator was used to estimate survival and differences in survival was assessed using the log-rank test. Hazard ratios (HR) for recurrence were estimated by univariable and multivariable Cox regression. The multivariable model included age, gender, HPV and radicality as covariables.

All tests were performed in SPSS (IBM Corp. , SPSS Statistics for Mac, Version 21.0. Armonk, NY, USA) and STATA (StataCorp, 4905 Lakeway Dr, College Station, TX, USA).

\section{Results}

98 of 126 IP from SCR were included in the study. Cases were 


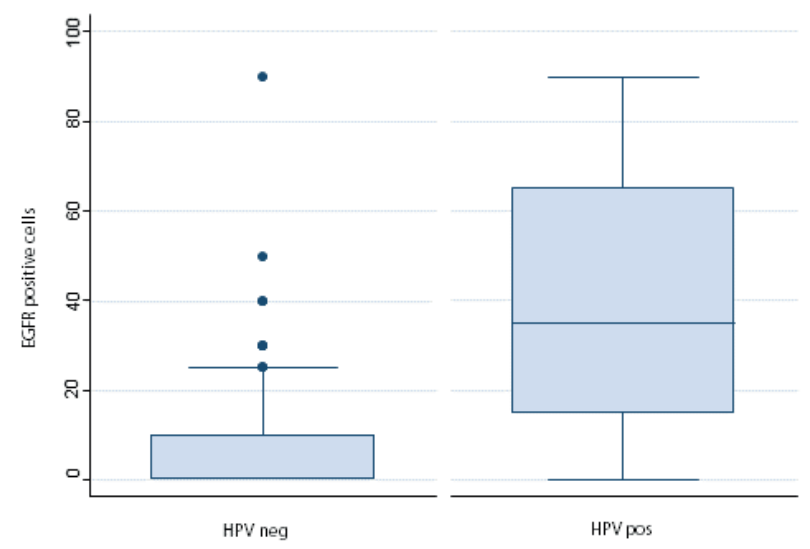

Figure 1. EGFR expression in correlation to HPV status.

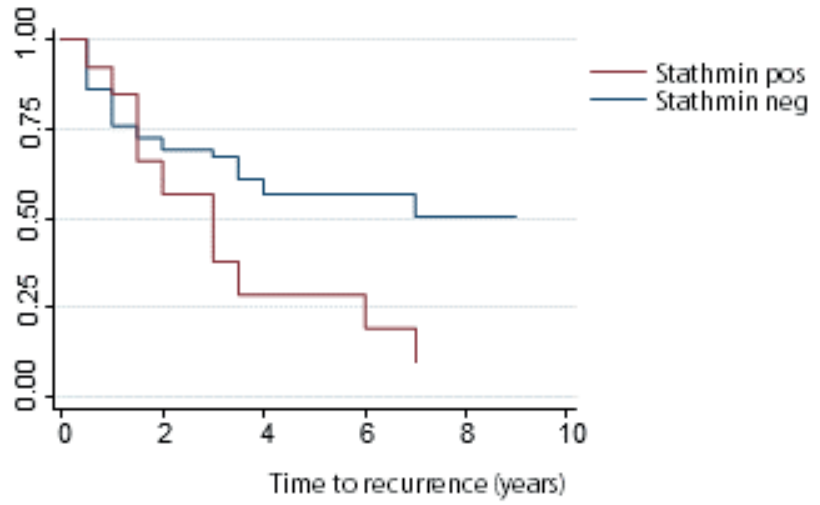

Figure 2. Kaplan-Meier survival estimates. excluded when re-evaluated as non-IP ( $n=6$ - in most cases exophytic papilloma), when diagnosis was unsure at re-evaluation $(n=8)$, when cases were missing or too little material was left to be representative of the tumour $(n=12)$ or when registered tumours were un-identifiable in the medical records or the SMB $(n=2)$. Excluded cases did not differ significantly from those included regarding sex and age at diagnosis (data not shown).

\section{HPV prevalence}

In total, 12 out of 98 patient samples (12.2\%) were HPV DNA positive, in which eleven were low-risk type positive, nine HPV-11 and two HPV-6 and one was high-risk HPV, HPV-4 (Table 1). Patients with HPV positive lesions were significantly younger (mean age 45.6 years vs. 59.6 years, $p=0.003$ ) and their lesions were reported more often to present with mild to moderate dysplasia (25.0\% vs. $8.1 \%$ ), but this difference was not significant $(p=0.1)$

\section{EGFR}

In all, $57.1 \%$ of the specimens expressed EGFR at any level and the range of EGFR expression was 0-90\%. A significantly higher proportion of the HPV-positive IPs expressed EGFR at any level as compared to the HPV-negative ones (91.7\% vs. $52.3 \%$, $\mathrm{p}=0.011$ ). HPV-positive tumours also had a significantly higher median proportion of EGFR-positive cells than the HPV-negative tumours (35\%, vs. $10 \%, p<0.010$, Figure 1). Age or gender did not influence the results. No significant association was seen between EGFR-positivity and recurrence (Table 2). IPs with dysplasia (in our material only light to moderate dysplasia) expressed EGFR at a similar level as those without dysplasia, with a median proportion of $10 \%(p=0.362)$.

\section{Stathmin}

Staining for stathmin failed in two specimens' slides. Stathmin expression was identified in all IPs and the range of stathmin positive tumour cells varied between $10-100 \%$, with a median expression of $50 \%$ positive tumour cells. Hence, stathmin expression was further categorized into low and high expression with the median value of $50 \%$ as a cut off. Therefore, in total, $15.5 \%$ of the tumours had high expression of stathmin.

Dysplasia was present in $26,7 \%$ of IPs with high stathmin expression while only $7.4 \%$ of IPs with low stathmin expression showed dysplasia ( $p=0,045)$. Also, significantly higher proportion of IPs with dysplasia had high expression of stathmin as compared to those without dysplasia ( $40.0 \%$ vs. $12.6 \%, p=0.045)$.

When analysing stathmin expression in relation to recurrence, patients with IPs having high expression of stathmin had earlier recurrences. However, this association lost statistical significance when including the time factor and censoring in the analyses (Table 3, log rank test: $p=0.053$, Figure 2 ).

No difference in level of stathmin expression was observed between HPV positive and negative IPs (27.3\% vs $30.2 \%$, $\mathrm{p}=0.25$ ). Likewise, there were no differences in levels of stathmin expression related to age or sex.

Besides tumour tissue, 73 of 96 slides also contained normal mucosa. In 23 of 73 slides (30.4\%) the mucosa did not express stathmin and in the remaining slides (61.6\%) the mucosa expressed stathmin very weakly, far from strong cytoplasmic staining which was the definition of positive stathmin staining.

\section{Discussion}

In this study, we found a significantly higher expression of EGFR in HPV positive tumours than in HPV negative tumours. We also report a significant association between high expression of stathmin and dysplasia and a tendency towards an association between stathmin and recurrence in IP.

Notably, EGFR-positivity was here defined in a similar way compared to what has previously been presented ${ }^{(13,17,18)}$ and like in previous studies, EGFR expression was higher in HPV positive IPs ${ }^{(13,18)}$. This might strengthen the previously stipulated hypothesis that there is a defined group of IPs where HPV infection is an early event among the different etiological factors that may 
Table 2. Clinical and histological data of the study population.

\begin{tabular}{|c|c|c|c|c|c|c|c|c|c|c|}
\hline & $\begin{array}{l}\text { Population } \\
\qquad \mathrm{N}=98\end{array}$ & $\begin{array}{l}\text { HPV+ } \\
n=12\end{array}$ & $\begin{array}{l}\text { HPV- } \\
n=86\end{array}$ & $\mathbf{p}$ & $\begin{array}{l}\text { EGFR+ } \\
n=56\end{array}$ & $\begin{array}{l}\text { EGFR- } \\
n=42\end{array}$ & $\mathbf{p}$ & $\begin{array}{l}\text { Stathmin+ } \\
\qquad n=15\end{array}$ & $\begin{array}{l}\text { Stathmin- } \\
\qquad \mathbf{n = 8 1}\end{array}$ & $\mathbf{p}$ \\
\hline \multicolumn{11}{|l|}{ Sex n (\%) } \\
\hline M & $68(69.4)$ & $9(13.2)$ & 59 (86.8) & & $41(60.3)$ & 27 (39.7) & & $10(15.2)$ & $56(84.8)$ & \\
\hline $\mathrm{F}$ & $30(30.6)$ & $3(10.0)$ & $27(90.0)$ & 0.69 & $15(50.0)$ & $15(50.0)$ & 0.34 & $5(16.7)$ & $25(83.3)$ & 0.83 \\
\hline \multicolumn{11}{|c|}{ Age at diagnosis } \\
\hline Median & 59.5 & 42.5 & 60 & & 58 & 60 & & 60 & 60 & \\
\hline Mean & 58.0 & 46.5 & 59.6 & & 58.4 & 59.6 & 0.06 & 62.5 & 59.8 & 0.68 \\
\hline Range & $18-89$ & $24-82$ & $18-89$ & 0.03 & $24-89$ & $18-85$ & & $29-85$ & $18-89$ & \\
\hline \multicolumn{11}{|l|}{ Radicality } \\
\hline Yes & $46(46.9)$ & $7(58.3)$ & $39(45.3)$ & & $28(50.0)$ & $18(42.9)$ & & $8(53.3)$ & $38(46.9)$ & \\
\hline No & $52(53.1)$ & $5(41.7)$ & $47(54.7)$ & 0.40 & $28(50.0)$ & $24(57.1)$ & 0.48 & $7(46.7)$ & $43(53.1)$ & 0.62 \\
\hline \multicolumn{11}{|c|}{ Recurrence } \\
\hline Yes & $40(40.8)$ & $4(33.3)$ & $36(41.9)$ & & 19 (33.9) & $21(50.0)$ & & $5(33.3)$ & $29(35.8)$ & \\
\hline No & $58(59.2)$ & $8(66.7)$ & $50(58.1)$ & 0.62 & $37(66.1)$ & $21(50.0)$ & 0.11 & $10(66.7)$ & $52(64.2)$ & 0.03 \\
\hline \multicolumn{11}{|l|}{ Dysplasia } \\
\hline Yes & $10(10.2)$ & $3(25.0)$ & $7(8.1)$ & & $7(12.5)$ & $3(7.1)$ & 0.39 & $4(26.7)$ & $6(7.5)$ & \\
\hline No & $88(89.8)$ & $9(75.0)$ & 79 (91.9) & 0.10 & 49 (87.5) & 39 ((92.9) & & $11(73.3)$ & 75 (92.5) & 0.02 \\
\hline
\end{tabular}

Table 3. Multivariate Cox regression.

\begin{tabular}{|llll|}
$\begin{array}{l}\text { Prognostic } \\
\text { factor }\end{array}$ & HR & $\begin{array}{c}\text { Confidence } \\
\text { Interval }\end{array}$ & p \\
\hline HPV & 0.49 & $0.17-1.38$ & 0.18 \\
\hline Radicality & 0.36 & $0.18-0.71$ & 0.03 \\
\hline Male gender & 1.15 & $0.59-2.24$ & 0.68 \\
\hline Age>50 & 0.75 & $0.40-1.42$ & 0.38 \\
\hline EGFR & 0.71 & $0.38-1.31$ & 0.27 \\
\hline Stathmin & 1.95 & $0.95-4.01$ & 0.07 \\
\hline
\end{tabular}

lead to a different entity of IPs by another pathophysiological pathway than HPV negative ones, possibly by activation of EGFR by the E5 protein ${ }^{(13)}$. It has been stipulated that the HPV E5 gene protein enhances the activation of EGFR ${ }^{(19,20)}$. In tumours harbouring low-risk HPV subtypes, which we mainly found $(11 / 12$ specimens), the E5 onco- protein is suggested to play an important role in oncogenic transformation, mainly by enhancing EGFR pathway signalling (21).

EGFR has previously been found to be higher in IPs with carcinomas than IPs without, although the results did not reach statistical significance ${ }^{(17)}$. Moreover, a study by Udager et al. found that HPV negative specimens demonstrated EGFR mutations and that possibly progression of IP's require overexpression or mutation of EGFR (21). EGFR- inhibitors are either tyrosine kinase inhibitors that bind to the tyrosine kinase domain in the EGFR, thereby inhibiting its activity or else monoclonal antibodies binding to the extracellular component of the EGFR thus

preventing epidermal growth factor from binding to its own receptor, therefore preventing cell division. EGFR inhibitors much used in the treatment of solid organ malignancies such as head and neck carcinoma. They are associated serious adverse effects, especially severe diarrhea and a range of cutaneous conditions ${ }^{(22-24)}$. It has also been suggested that EGFR-inhibitors could be tested in low-risk HPV positive IP's ${ }^{(18)}$. Considering the costs and side-effects of such treatment one should probably be more restrictive and reserve administration of EGFR-inhibitors to patients with EGFR-positive IPs with malignant transformation or extra-sinonasal growth where radical surgery would be too mutilating. Irreversible EGFR-inhibitors have previously been tested in vitro on SCC derived from IPs with and without mutated EGFR genes with promising results ${ }^{(22)}$.

Nonetheless, no association between EGFR expression and dysplasia was observed. To our knowledge, few studies have addressed this issue. A study by Katori et al, reported increased EGFR expression in premalignant and malignant tumours as compared to normal mucosa and IPs with mild to moderate dysplasia. However, no comparison to IPs without dysplasia was performed. In our study, no IP with severe dysplasia was included. We did not find an association between expression of EGFR and recurrence. Obviously, more studies on EGFR expression in IP is needed to investigate its clinical role in IP.

In line with a previous study by Lin et al, we found that stathmin was overexpressed in IP as compared to the normal mucosa, which did not express or expressed stathmin weakly ${ }^{(8)}$. 
Furthermore, high expression of stathmin was overrepresented among specimens with dysplasia and there was a tendency for the same pattern in cases with recurrence. These results are also similar to what has been presented by others in malignant tumours, where a high stathmin expression is correlated to a poorer prognosis ${ }^{(23)}$. If these results can be reproduced, stathmin may be a plausible marker of prognosis or a treatment target for recurring, surgically challenging cases.

In our material, $27 \%$ of the HPV positive IPs were stathmin positive. The number of positive specimens is, of course related to the level set as positive. For stathmin, this has previously been managed in different ways. Most studies have presented a defined stathmin positivity and negativity level instead of presenting the fraction of positive cells. However, the cut-off level between stathmin positivity and stathmin negativity has varied ${ }^{(24-26)}$. We chose a cut-off point at $50 \%$ as previously has been done because it was a level that had previously been used ${ }^{(6,7)}$. Interestingly, on slides containing IP with surrounding normal mucosa, the normal sinonasal mucosa did not at all express, or expressed stathmin with a low staining intensity. This suggests that stathmin is expressed more strongly in IP than in normal mucosa. Possibly IPs could therefore be treated by stathmin targeted therapy in the future if these results can be confirmed by other studies.

\section{Conclusion}

In conclusion, EGFR expression is significantly higher in HPV positive than HPV negative specimens and EGFR could possibly be a therapeutic target in EGFR positive tumours.

Stathmin is consistently expressed in inverted papillomas but not in the normal, non -neoplastic mucosa. Stathmin positivity seems to be associated with dysplasia and possibly also with recurrence. Stathmin might therefore even more than EGFR be considered a therapeutic target.

\section{Acknowledgements}

Financial support provided by: The ACTA Otolaryngologica Foundation, The Swedish Society of Medicine and the Nachmanson Foundation through The Swedish ENT Association for Otorhinolaryngology, Head and Neck surgery (SFOHH).

\section{Authorship contribution}

AE: Study design, collect patient data, laboratory analysis, statistical analysis, manuscript writing, corresponding author. AN: study design, responsible for laboratory methods, laboratory analysis, manuscript revision. LHN: study design, manuscript revision. MW: statistical analysis, manuscript revision. PS: study design, manuscript revision. LM: study design, valuating laboratory results, manuscript writing and revision.

The study includes more than 5 authors since Marit Westman was brought in to the study group for her epidemiological and statistical experience to sharpen the statistics in the study.

\section{Conflict of interest}

No conflict of interest.

\section{References}

1. Elliot A, Marklund L, Håkansson $\mathrm{N}$ et al. Incidence of IP and risk of malignant transformation in the Swedish population 1960 2010. Eur Arch Otorhinolaryngol 2017; 274 1445-1448.

2. Suh JD, Ramakrishnan VR, Thompson CF, et al. Inverted papilloma of the sphenoid sinus: risk factors for disease recurrence. Laryngoscope 2015; 125(3): 544-8.

3. Syrjanen $\mathrm{K}$ and Syrjanen S. Detection of human papillomavirus in sinonasal papillomas: systematic review and meta-analysis. Laryngoscope 2013; 123: 181-92.

4. Rubin $\mathrm{Cl}$ and Atweh GF. The role of stathmin in the regulation of the cell cycle. J Cell Biochem 2004; 93: 242-50.

5. Kouzu Y, Uzawa K, Koike et al Overexpression of stathmin in oral squamous-cell carcinoma: correlation with tumour progression and poor prognosis. $\mathrm{Br}$ J Cancer 2006; 94: 717-23.

6. Lin WC, Chen SC, Hu FC et al. Expression of stathmin in localized upper urinary tract urothelial carcinoma: correlations with prognosis. Urology 2009; 74: 1264-9.

7. Suzuki S, Yokobori T, Altan B et al. High stathmin 1 expression is associated with poor prognosis and chemoradiation resistance in esophageal squamous cell carcinoma. Int J Oncol; 2017.

8. Lin H, Lin D and Xiong XS. Roles of human papillomavirus infection and stathmin in the pathogenesis of sinonasal inverted papilloma. Head Neck 2016; 38: 220-4

9. Park HS, Jang MH, Kim EJ et al. High EGFR gene copy number predicts poor outcome in triple-negative breast cancer. Mod Pathol 2014; 27: 1212-22.

10. Veale D, Ashcroft T, Marsh C and Harris AL. Epidermal growth factor receptors in nonsmall cell lung cancer. Br J Cancer 1987; 55: 513-6.

11. Miyaguchi M, Sakai S, Olofsson J, Kuwabara $\mathrm{H}$ and Sakamoto $\mathrm{H}$. Prognostic significance of epidermal growth factor receptor in squamous cell carcinoma of the maxillary sinus. Eur Arch Otorhinolaryngol 1993; 249: 478-81.

12. Ford AC and JR Grandis. Targeting epidermal growth factor receptor in head and neck cancer. Head Neck 2003; 25: 67-73.

13. Katori H, Nozawa A and Tsukuda M. Markers of malignant transformation of sinonasal inverted papilloma. Eur J Surg Oncol 2005; 31: 905-11.
14. Chao JC and Fang SY. Expression of epidermal growth factor receptor in the inverted papilloma and squamous cell carcinoma of nasal cavity. Eur Arch Otorhinolaryngol 2008; 265: 917-22.

15. Dalianis T, Grün N, Koch J et al. Human papillomavirus DNA and p16(INK4a) expression in hypopharyngeal cancer and in relation to clinical outcome, in Stockholm, Sweden. Oral Oncol 2015; 51: 857-61.

16. Marklund L, Näsman A, Ramqvist T, Dalianis $T$, Munck-Wikland E and Hammarstedt L. Prevalence of human papillomavirus and survival in oropharyngeal cancer other than tonsil or base of tongue cancer. Cancer Med 2012; 1: 82-8.

17. Lin GC, Scheel A, Akkina S et al. Epidermal growth factor receptor, p16, cyclin D1, and p53 staining patterns for inverted papilloma. Int Forum Allergy Rhinol 2013; 3: 885-9.

18. Scheel A, Lin GC, McHugh JB et al. Human papillomavirus infection and biomarkers in sinonasal inverted papillomas: clinical significance and molecular mechanisms. Int Forum Allergy Rhinol 2015; 5: 701-7.

19. Venuti A, Paolini F, Nasir L et al. Papillomavirus E5: the smallest oncoprotein with many functions. Mol Cancer 2011; 10: 
140.

20. Wechsler El, Tugizov S, Herrera R, Da Costa $\mathrm{M}$ and Palefsky JM. E5 can be expressed in anal cancer and leads to epidermal growth factor receptor-induced invasion in a human papillomavirus 16-transformed anal epithelial cell line. J Gen Virol 2018; 99 : 631-644.

21. Udager AM, MCHugh JB, Goudsmit CM et al. Human papillomavirus (HPV) and somatic EGFR mutations are essential, mutually exclusive oncogenic mechanisms for inverted sinonasal papillomas and associated sinonasal squamous cell carcinomas. Ann Oncol 2018; 29: 466-471.

22. Expert Opin Investig Drugs. Indian Dermatol Online J. 2019; $10: 251-255$.

23. Matos I, Noguerido A, Ros J et al. Triple-drug chemotherapy regimens in combination with an anti-EGFR agent in metastatic colorectal cancer - prospects from phase II clinical trials. Expert Opin Investig Drugs. 2019; 28: 463-471.

24. Saraswat N, Sood A, Kumar D et al. Clinical Profile of Cutaneous Adverse Effects of Epidermal Growth Factor Receptor
Inhibitors: A Prospective Observational Study of 76 Cases. Indian Dermatol Online J. 2019;10:251-255.

25. Udager AM, Rolland DCM, MCHugh JB et al. High-Frequency Targetable EGFR Mutations in Sinonasal Squamous Cell Carcinomas Arising from Inverted Sinonasal Papilloma. Cancer Res 2015:75: 2600-2606.

26. Ni PZ, He JZ, Wu ZY et al. Overexpression of Stathmin 1 correlates with poor prognosis and promotes cell migration and proliferation in oesophageal squamous cell carcinoma. Oncol Rep 2017; 38: 3608-3618

27. Hemdan $T$, Lindén $M$, Lind SB et al. The prognostic value and therapeutic target role of stathmin-1 in urinary bladder cancer. Br J Cancer 2014; 111:1180-7.

28. Novak M, Lester J, Karst AM et al. Stathmin 1 and p16(INK4A) are sensitive adjunct biomarkers for serous tubal intraepithelial carcinoma. Gynecol Oncol 2015; 139: 104-11.

29. Chen Y, Zhang Q, Ding C, Zhang X, Qiu X and Zhang Z. Stathmin 1 overexpression in hypopharyngeal squamous cell carcinoma: A new promoter in $\mathrm{FaDu}$ cell proliferation and migration. Int J Oncol 2017; 50: 31-40.

\section{Alexandra Elliot, MD, PhD \\ Department of Clinical Science \\ Intervention and Technology \\ Division of ENT Diseases \\ Karolinska Institutet \\ Stockholm \\ Sweden}

Department of Head and Neck

Lung and Skin Cancer

Karolinska University Hospital

17176 Stockholm

Sweden

Tel: +46 $851776126,+46704334635$

E-mail: Alexandra.elliot@sll.se 